Part of Journal of Research of the National Bureau of Standards, Volume 29, December 1942

\title{
INTERVAL SELECTOR FOR RANDOM PULSES
}

\author{
By Francis J. Davis and Leon F. Curtiss
}

\section{ABSTRACT}

An interval selector is described which has been developed to study the distribution of pulses from Geiger-Müller counters. The circuit is designed to count pulses with a separation less than $\tau$, where $\tau$ may be varied between $3 \times 10^{-5}$ sec and 0.2 sec. Tests are described which show that the circuit accomplishes these measurements with considerable precision.

Results of tests on alcohol-vapor-argon and amyl-acetate-vapor-argon-type Geiger-Müller counters are given which show that counters of this type can be prepared in a simple manner, yielding the proper random distribution of pulses even at very rapid rates of counting. Counters thus prepared have maintained their characteristics for at least 2 years.

\section{CONTENTS}

I. Introduction

II. Interval selector

III. Test of alcohol-argon-type counters

\section{INTRODUCTION}

The increasing use of Geiger-Müller tube counters for quantitative measurements under widely varying conditions has led to a need for methods to test these counters in a more discriminating manner than has previously been customary. It is no longer sufficient that a counter have a "plateau" sensibly horizontal and of an extent to make the counter convenient to use. For reliable measurements it is essential that all pulses arise in the counter in response to radiation. Spurious pulses, frequently associated with the true pulses as one or more after-pulses, are one of the defects in counters which make them unreliable for quantitative work. Such defects can be detected by a study of the distribution of the intervals between pulses. If these intervals follow Poisson's equation for a random distribution, it is generally assumed that the counter is satisfactory. Conversely, a counter is definitely unreliable if it does not satisfy this requirement.

An examination of the distribution of pulses from a counter with respect to time is likely to be very laborious in the absence of some device to determine automatically the number of pulses with a separation, for example, less than some selected interval. For reasonably accurate information, thousands of pulses must be counted for each interval selected. Recently two different circuits have been proposed 
to permit automatic counting of intervals, one by Driscoll, Hodge, and Ruark ${ }^{1}$ and the other by Roberts. ${ }^{2}$ The former circuit determines the number of intervals longer than a chosen interval. A relay incorporated in this circuit limits its use to intervals longer than about 0.1 sec.

For the study of multiple pulses, it is much more convenient to determine the number of intervals shorter than a chosen interval $\tau$ and also to be able to choose $\tau$ to have any value from the order of 1 sec down to the resolving time of the tube counter under investigation. The circuit described by Roberts (see footnote 2 ) operates in this manner and is capable of choosing intervals of trom $10^{-4}$ sec to $0.3 \mathrm{sec}$. However, this circuit is somewhat more complicated than necessary, making adjustments difficult to secure the desired precision.

The circuit described below is of a different design, which has shown itself to be an improvement over either of those mentioned above. It is capable of selecting intervals as short as $3 \times 10^{-5} \mathrm{sec}$ with considerable precision.

\section{INTERVAL SELECTOR}

The diagrams for this interval selector are shown in figures 1 and 2 . The negative pulses from the Geiger counter to be tested are fed to the preamplifier, which is a twin-triode, $T_{1}$ and $T_{2}$, connected in the usual capacity-coupled manner. The preamplifier renders the apparatus sensitive to minimum pulses of about 1 volt at the counter center wire.

The next two tubes, $T_{3}$ and twin-triode $T_{4}$ and $T_{5}$, comprise a pulse leveler-sharpener. Its action rejects all pulses below the original 1-volt minimum and gives a constant output pulse for each pulse over 1 volt. The second triode, $T_{5}$, of the $6 \mathrm{SC} 7$ is used simply to invert the negative pulses to positive pulses.

Normally $T_{3}$ is conducting and the triode $T_{4}$ nonconducting. If a small pulse is introduced at the grid of $T_{3}$, it is amplified at the plate and fed to the grid of $T_{4}$; if this amplified pulse is not large enough to bring the grid to the cut-off potential, which is about -4 volts, then that is as far as the pulse goes. If the pulse is large enough, it is amplified by the triode $T_{4}$ and applied to the injector grid of $T_{3}$, which
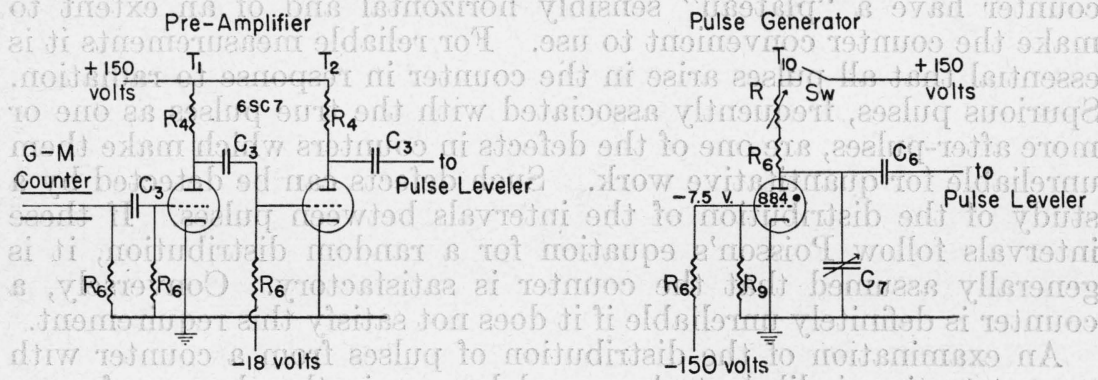

Figure 1.-Circuit diagram of preamplifier and pulse generator.

$C_{7}$ is a bank of condensers attached to an 11-point switch.

$$
\begin{array}{ll}
R=1 \mathrm{meg} o \mathrm{hm} & C_{6}=35 \mu \mu \mathrm{f} \\
R_{9}=5,000 \mathrm{ohms} & C_{7}=0.0001 \text { to } 0.5 \mu \mathrm{f}
\end{array}
$$

1 R. L. Driscoll, Mary W. Hodge, and A. E. Ruark, Rev. Sci. Instr. 11, 241 (1940).
2 A. Roberts, Rev. Sci. Inst. 12, 71 (1941). 


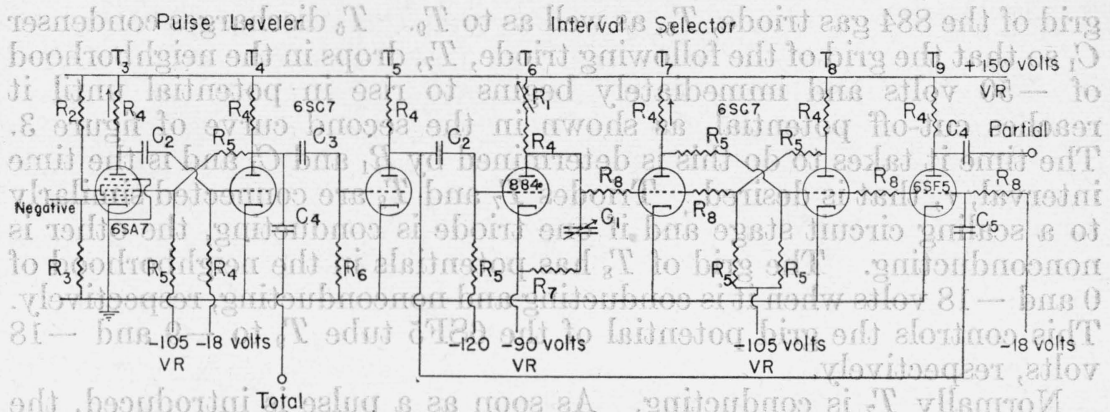

FIGURE 2.-Circuit diagram of pulse leveler and interval selector.

$C_{1}$ is a bank of condensers attached to an 11-point switch. Voltages marked $V R$ are controlled by $V R$ type voltage regulator tubes.

$$
\begin{array}{lll}
R_{1}, R_{3}=1 \text { megohm } & C_{1}=0.0005 \text { to } 2 \mu \mathrm{f} \\
R_{2}=25,000 \mathrm{ohms} & C_{2}=100 \mu \mu \mathrm{f} \\
R_{4} \quad=50,000 \mathrm{ohms} & C_{3}=50 \mu \mu \mathrm{f} \\
R_{5} \quad=250,000 \mathrm{ohms} & C_{4}=200 \mu \mu \mathrm{f} \\
R_{6}=100,000 \mathrm{ohms} & C_{5}=10 \mu \mu \mathrm{f} \\
R_{7}=300 \mathrm{ohms} & & =0 \\
R_{8} & =0.5 \text { megohm } &
\end{array}
$$

further amplifies it, and this regeneration continues until $T_{3}$ is nonconducting and $T_{4}$ conducting. Since the plate voltage of $T_{3}$ is no longer changing, the charge leaks off the grid of $T_{4}$, returning $T_{4}$ to the nonconducting state. In this manner it is seen that the output pulses will be the same height, since either a pulse will be rejected at $T_{4}$ or amplified until $T_{3}$ is nonconducting and $T_{4}$ conducting, giving a pulse of full voltage. The width of the pulses from the pulse leveler will also be constant because that is determined by the $R C$ constants and bias potential of the $T_{4}$ grid. If a wide pulse is fed into $T_{3}$, this stage is held nonconducting beyond the time $T_{4}$ becomes nonconducting. If it is a very narrow pulse, $T_{3}$ is held nonconducting by the injector grid until $T_{4}$ becomes nonconducting. For the constants used, the pulse leveler has a resolving time of the order of $3 \times 10^{-5}$ sec. In order to have a short resolving time, one must sacrifice sensitivity, therefore making the preamplifier necessary.

The first curve shown in figure 3 represents the negative pulses occurring at the grid of $T_{5}$. These pulses are inverted and fed to the

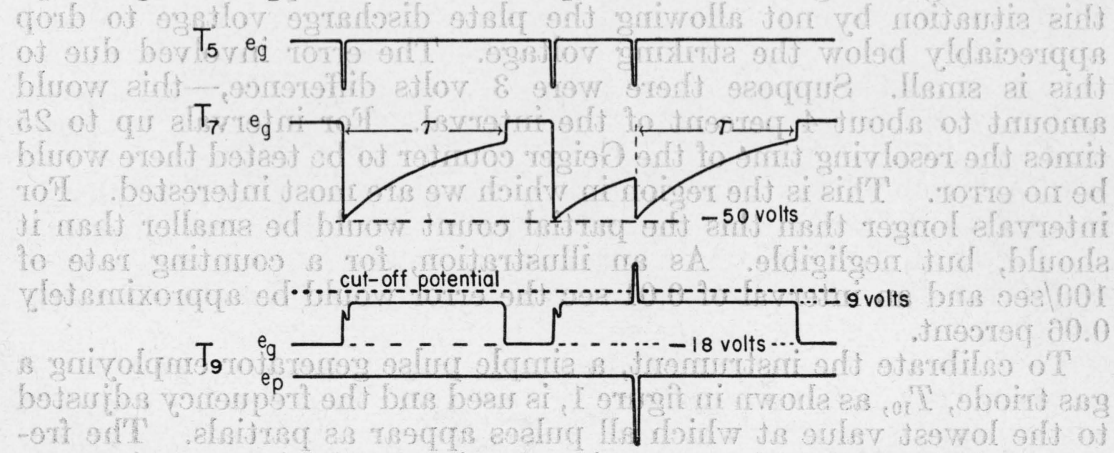

FIGURE 3.-Diagram of corresponding voltages in different parts of the circuit.

The distribution of pulses is assumed as shown on the $T_{5}$ grid. $\tau$ is the chosen interval; $e_{\mathrm{g}}$ and $e_{p}$ are the grid and plate potentials, respectively. Pulses appearing on plate $T_{9}$ are exclusively those which follow a preceding pulse at an interval less than $\tau$. 
grid of the 884 gas triode, $T_{6}$, as well as to $T_{9} . \quad T_{6}$ discharges condenser $C_{1}$ so that the grid of the following triode, $T_{7}$, drops in the neighborhood of -50 volts and immediately begins to rise in potential until it reaches cut-off potential, as shown in the second curve of figure 3. The time it takes to do this is determined by $R_{1}$ and $C_{1}$ and is the time interval, $\tau$, that is desired. Triodes $T_{7}$ and $T_{8}$ are connected similarly to a scaling circuit stage and if one triode is conducting, the other is nonconducting. The grid of $T_{8}$ has potentials in the neighborhood of 0 and -18 volts when it is conducting and nonconducting, respectively. This controls the grid potential of the 6 SF5 tube $T_{9}$ to -9 and -18 volts, respectively.

Normally $T_{7}$ is conducting. As soon as a pulse is introduced, the gas triode, $\mathrm{T}_{6}$, discharges condenser $C_{1}$ so that the grid of $T_{7}$ is below cut-off potential and nonconducting. This renders $T_{8}$ conducting, which brings $T_{9}$ grid potential to -9 volts, as shown in the third curve of figure 3 , so that if a second pulse comes within the chosen interval (the third pulse shown in fig. 3 is an example), it will be amplified by $T_{9}$, as shown by the fourth curve in figure 3 , and registered on the partial recorder. The gas triode $T_{6}$ discharges $C_{1}$ on each pulse, so that another interval is started. If the second pulse does not fall within the prescribed interval (the second pulse shown in fig. 3 is an example), $T_{7}$ becomes conducting, $T_{8}$ nonconducting, the grid potential of $T_{9}$ falls back to -18 volts, and therefore the pulse is not amplified and registered on the partial recorder. The 0.5 megohm resistors on the grid of $T_{7}$ prevent oscillations on long-time intervals when the grid of $T_{7}$ would be brought slowly through cut-off potential.

A gas triode is used for $T_{6}$ to discharge condenser $C_{1}$ completely and rapidly, independently of the input pulse. If a hard vacuum tube were used, the plate current discharging the condenser would depend on the plate voltage and the input pulse width and voltage. For the narrow pulses used, condenser $C_{1}$ for long intervals and necessarily large capacity would not become completely discharged. The 884 is not without its disadvantages, however. The striking voltage of a gas tube is higher than its glow voltage. If two pulses fall very close together, compared to the time interval, the second pulse will not strike the 884 , since the plate voltage has not had time to rise beyond the striking voltage. The $300-\mathrm{ohm}$ resistor in the plate circuit helps this situation by not allowing the plate discharge voltage to drop appreciably below the striking voltage. The error involved due to this is small. Suppose there were 3 volts difference,- - this would amount to about 4 percent of the interval. For intervals up to 25 times the resolving time of the Geiger counter to be tested there would be no error. This is the region in which we are most interested. For intervals longer than this the partial count would be smaller than it should, but negligible. As an illustration, for a counting rate of $100 / \mathrm{sec}$ and an interval of $0.01 \mathrm{sec}$ the error would be approximately 0.06 percent.

To calibrate the instrument, a simple pulse generator employing a gas triode, $T_{10}$, as shown in figure 1 , is used and the frequency adjusted to the lowest value at which all pulses appear as partials. The frequency is determined by measuring the time to obtain a certain number of pulses. This "pulse count" was obtained by the two scaling circuits used to determine the partial and total count. Each scaling circuit has a scale of 128 , and when connected in series they give a 
scale of 16,384 . Voltage regulators are used throughout, so that the calibration for a given setting of $R_{1}$ and $C_{1}$ remains constant,

For the constants used the range of intervals obtainable is between $3 \times 10^{-5}$ and $0.2 \mathrm{sec}$. For most counters, that is, those having a resolving time greater than $3 \times 10^{-5} \mathrm{sec}$, no correction is necessary for the resolving time of instrument.

The symbols used are the same as Roberts', namely:

$t=$ resolving time of counter.

$\tau=$ time interval.

$N_{\text {exp }}=$ Number of partial counts per second (intervals per second less than $\tau$ ) measured experimentally.

$a=$ total number of counts per second measured experimentally.

$A=$ theoretical true count, assuming $a=A e^{-A t}$ and resolving time $t$.

$N_{\mathrm{th}}=a\left(1-e^{-a \tau}\right)$, theoretical number of intervals per second less than $\tau$, assuming $a$ as the true count per second.

$N=A\left(e^{-A t}-e^{-A \tau}\right)=a-A e^{-A \tau}=$ theoretical number of intervals per second less than $\tau$ and greater than $t$, assuming $A$ true counts per second $(\tau>t)$.

From the last equation we obtain $\ln (a-N)=\ln A-A \tau$, so that plotting $\ln (a-N)$ against $\tau$ gives a straight line of slope $-A$ for $\tau>t$, which extrapolated to $\tau=0$ gives $\ln A$. A necessary but not sufficient condition is that in plotting $\ln \left(a-N_{\text {exp }}\right)$ against $\tau$ we obtain a straight line. The log of the negative of the slope must also equal the intercept. A method of investigating this condition is to compare values of $t$ obtained by (1) finding the value of $\tau$ on the graph corresponding to $1 \mathrm{n} a$ and (2) determine $A$ from the intercept and $t$ from $a=A e^{-A t}$.

A defect in certain counters is a tendency to produce multiple pulses. This defect can be shown to better advantage by plotting $N_{\text {exp }} / N_{\text {th }}$ against $\tau$. For short intervals the curve should approach zero as we approach the resolving time of the counter, and for long intervals the curve should approach unity, as in figures 5, 7, and 11 . The experimental points are shown by the circles, and the solid line is the theoretical curve $N / N_{\text {th }}$.

As a test of the reliability of the instrument, alpha particles from a natural radioactive substance of long life, in equilibrium with its decay products in a parallel plate ionization chamber with a linear amplifier, were used as a random source.

The results are shown in figure 4 and 5 . The experimental points are noticed to be a closer fit to the theoretical curves than in the following curves. This is not due entirely to a better source, but also more care was taken to reduce statistical error by obtaining higher counts of partial and total intervals. For short intervals appreciable time must elapse to obtain a considerable count, and where several counters are being tested for possible multiple pulses, such accuracy is not needed. A slightly different method of calculating the theoretical curves was used in this case, to get a better fit. In the following curves, $A$ is determined by the intercept of the straight line fitted to the data shown in graphs similar to figure 4 and this value of $A$ used to calculate the theoretical curve in the graphs similar to figure 5 . In the present case, however, $A$ was chosen to give a theoretical curve of best fit in figure 5 and this value of $A$ used as its intercept in figure 4 . 
on The value of the resolving time, $t$, calculated from $A$ is $3.74 \times 10^{-4}$ sec. The value of $t$ obtained from figure 4 is $(3.7 \pm 0.1) \times 10^{-4}$ sec. The $\pm 0.1 \times 10^{-4}$ is roughly estimated from the permissible shift in the slope of the line in figure 4 and still retain an apparently good fit. It might be asked why a least-square method of fitting is not used. In the first place, since the function is not algebraic, it involves considerable computation; and second, since the points representing the

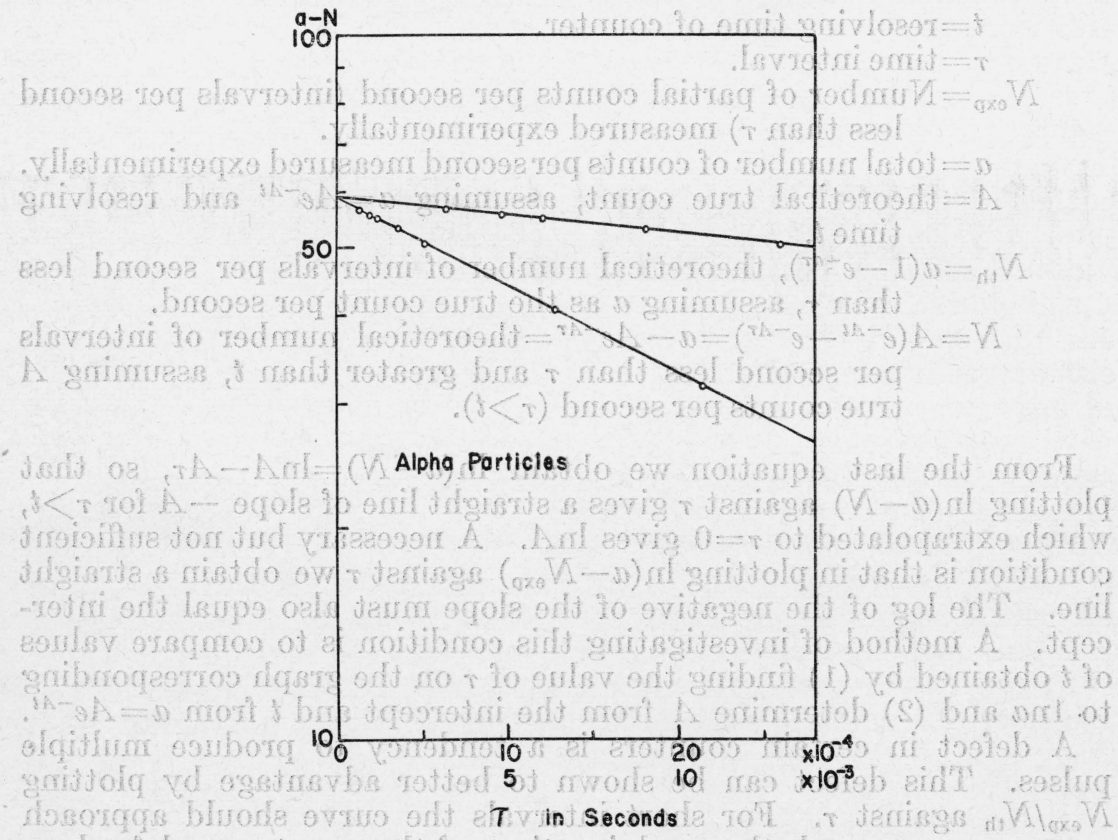

FIGURE 4. Interval data taken with an ionization chamber and source of alpha

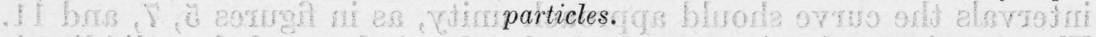
$a-N$ is plotted on a logarithmie soale against $\tau$. The graphs are straight lines drawn through the experi mental points. The two lines represent the same data plotted to the two scales shown for the $\tau$ axis.

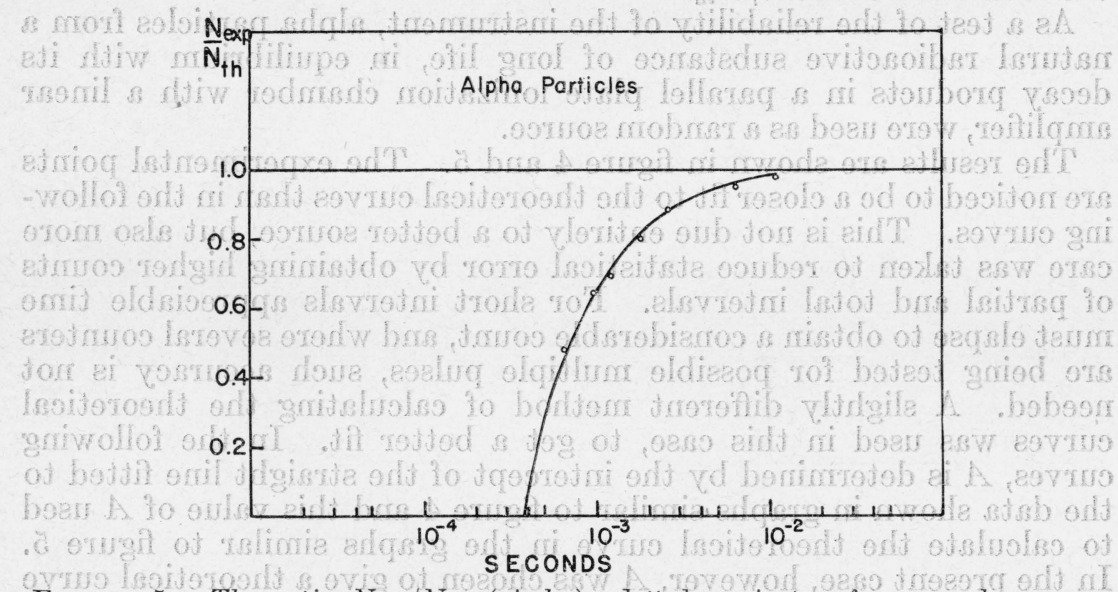

FiguRE 5. The ratio $N_{\text {exp }} / N_{\text {th }}$ (circles) plotted against $\tau$ for a random source. The data shown is obtained from alpha particles counted by an ionization chamber. The solid curve is calculated as described in the text. 
short intervals are more important in determining resolving time and showing of multiple pulses, these points probably should be weighted and this introduces certain arbitrariness Graphs of the type in figure 4 show errors in partial counts of long intervals to better advantage, whereas graphs of the type in figure 5 , since they approach unity for long intervals, show errors better in partial counts of short intervals. The lower end of the theoretical curve in figure 5 is shifted considerably to the right or left for small changes in the choice of $A$, and for this reason a more accurate determination of $A$ is obtained by fitting the curve in figure 5 . However, when one is not so much interested in the accuracy of $A$, it is much simpler to take $A$ as the intercept from the curves of the type shown in figure 5 , as was done in the following curves.

\section{TEST OF ALCOHOL-ARGON-TYPE COUNTERS}

The cireuit deseribed above was constructed to test the behavior of alcohol-argon-type counters to which organic vapors have been added. The most familiar combination is the alcohol-argon mixture, which was the first to be used for this purpose. The outstanding advantage of these counters is their self-quenching property, which enables them to respond to relatively rapid successions of pulses. They are not dependent on auxiliary quenching circuits, which are not always reliable, However, unless properly prepared they may have poor characteristics which limit their use. The results described below show that it is possible to prepare counters with organic vapors which show the expected distribution of intervals with great fidelity.

Figure 6 shows an alcohol-argon counter which satisfies these

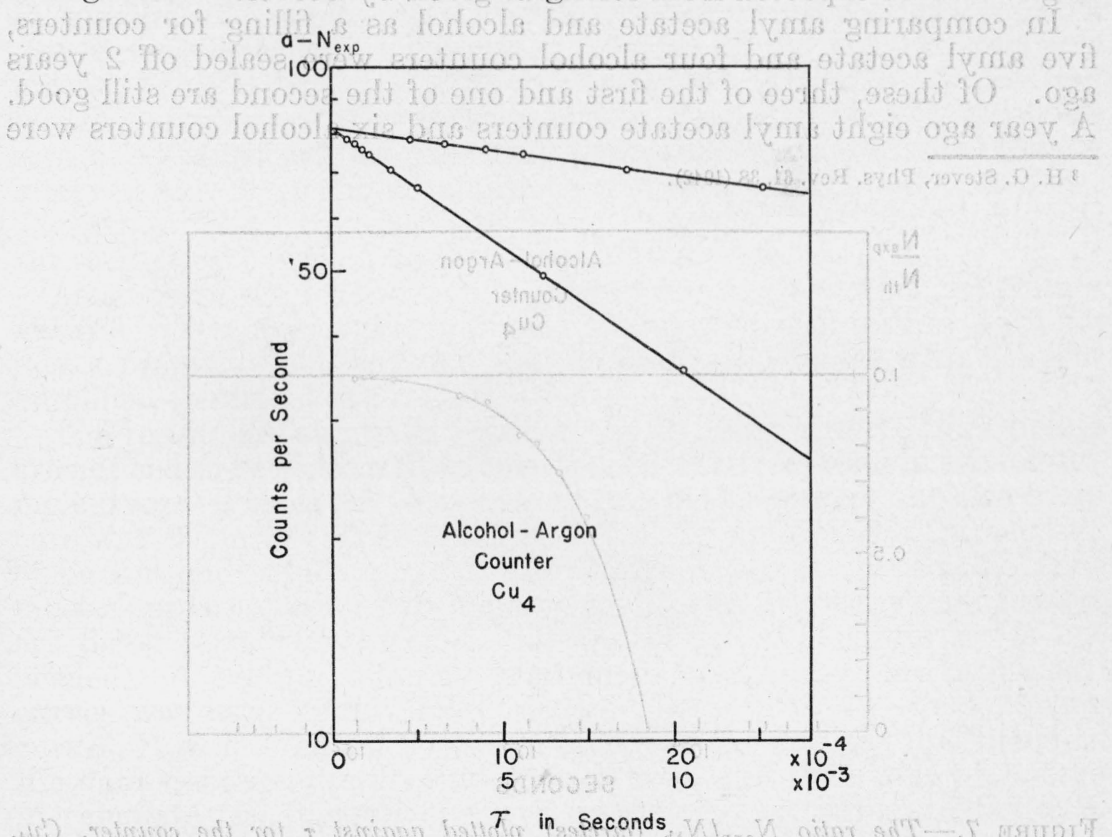

FIGURE 6.-Interval data taken with an alcohol-argon $C u_{4}$ counter.

$a-N_{\text {exp }}$ is plotted on a logarithmic scale against $\tau$. The graphs are straight lines drawn through the experimental points. The two lines represent the same data plotted to the two scales shown for the $\tau$ axis. 
conditions quite well. Here we have plotted $\ln \left(a-N_{\text {exp }}\right)$ against $\tau$ in seconds, which affords us two ways of finding $t$, as mentioned at the end of the preceding section. The two methods of finding $t$ give $1.90 \times 10^{-4} \mathrm{sec}$ and $1.95 \times 10^{-4} \mathrm{sec}$. The two lines are the same data, the upper line merely being the shorter time intervals spread on a larger scale.

Figure 8 shows a defective alcohol-argon counter, giving too many short intervals, probably due to multiple pulses. Roberts gives further examples. However, not all alcohol-argon counters show this defect, as you might be tempted to believe from his paper. Figure 7 is an example. There seems to be a correlation in that this defect is apt to show up in counters having a short voltage range of high slope, as shown in figure 9 .

Amyl acetate and argon seem to be a more reliable filling for counters. Typical curves are figures 10 and 11, showing a long voltage range of low slope and no obvious multiple pulses. Possibly a reason would be that amyl acetate being a heavier molecule has a higher quenching action. The higher resolving time indicated by the intercept on the $\tau$ axis of figure 11 is probably due mostly to the differences in the diameters of the counters used. The counters in figures 8 and 11 had diameters of 1.7 and $4.8 \mathrm{~cm}$, respectively.

Measurements made on about 30 different counters of various fillings of amyl acetate and alcohol with argon show that for a counter diameter of $2 \mathrm{~cm}$ the resolving time may vary from about $1.5 \times 10^{-4}$ to $4 \times 10^{-4}$ sec, for a 5 -cm counter $3 \times 10^{-4}$ to $7 \times 10^{-4} \mathrm{sec}$, and for a 10 -cm counter $1.5 \times 10^{-3}$ to $3 \times 10^{-3}$ sec. Although an exact formula for resolving time has not been worked out, a relation of this sort might well be expected from formulas given by Stever. ${ }^{3}$

In comparing amyl acetate and alcohol as a filling for counters, five amyl acetate and four alcohol counters were sealed off 2 years ago. Of these, three of the first and one of the second are still good. A year ago eight amyl acetate counters and six alcohol counters were

${ }^{3}$ H. G. Stever, Phys. Rev. 61, 38 (1942).

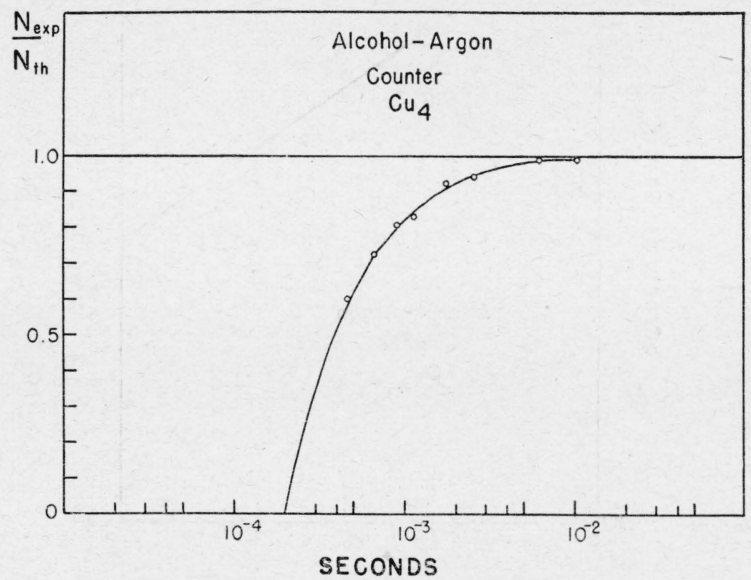

Figure 7.-The ratio $N_{\text {exp }} / N_{\text {th }}$ (circles) plotted against $\boldsymbol{\tau}$ for the counter, $C u_{4}$.

The theoretical curve for $N / N_{\text {th }}$ has been drawn in showing very good agreement. The intersection of this curve with the $\tau$ axis gives the resolving time of the counter. 
sealed off. Of these, four amyl acetate counters are still good but none of the alcohol type can be used.

A difficulty encountered in filling counters is the slow diffusion rate of the gases. -A counter filled by usual methods of letting in amyl acetate and argon and watching on an oscilloscope until it seems to be working at its best may work beautifully immediately after it has

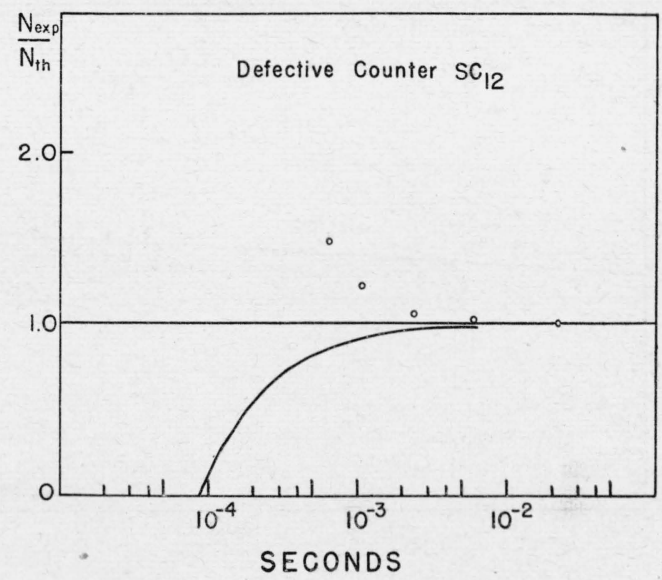

Figure 8.-Data from a defective counter, $S C_{12}$.

Too many short intervals are shown, due probably to multiple or spurious pulses.

been sealed off, but several hours later it may be useless, or have a short voltage range of high slope.

An experiment was tried with an aluminum counter which was giving just such trouble. A small capsule of amyl acetate, together with a metal plunger, was put into a bulb attached to the counter by about $15 \mathrm{~cm}$ of $10-\mathrm{mm}$ glass tubing. The counter was then filled with $8 \mathrm{~cm}$ of argon, sealed off, and the metal plunger shaken until

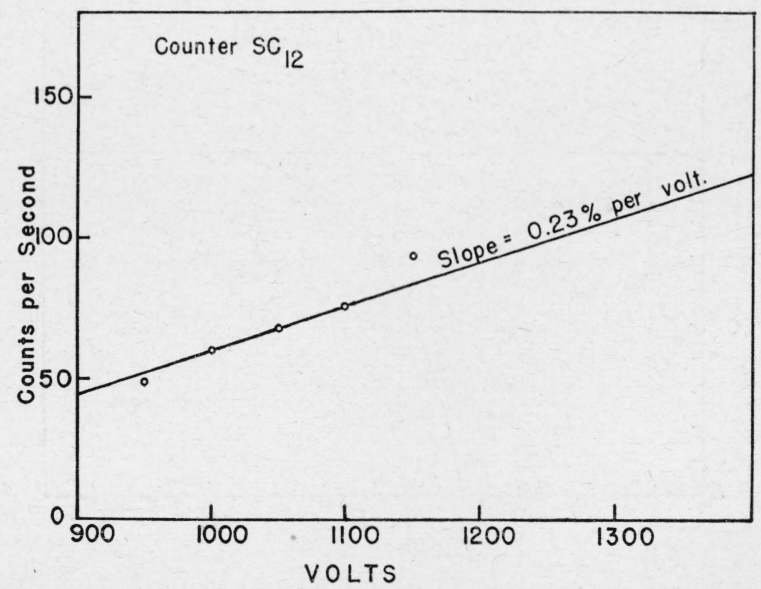

Figdre 9.-Voltage characteristic of the defective counter, $S C_{13}$.

A plateau of short range and excessive slope is shown. 
the capsule of amyl acetate was broken. Very shortly the voltage range was run, giving a plateau of low slope beginning at about 1,000 volts. A day later the counter would not operate below 1,500 volts at room temperature. The counter was then placed in an ice bath and after about one-half hour the voltage range was again run, showing a very nice plateau with a slope of about 0.03 percent per volt be-

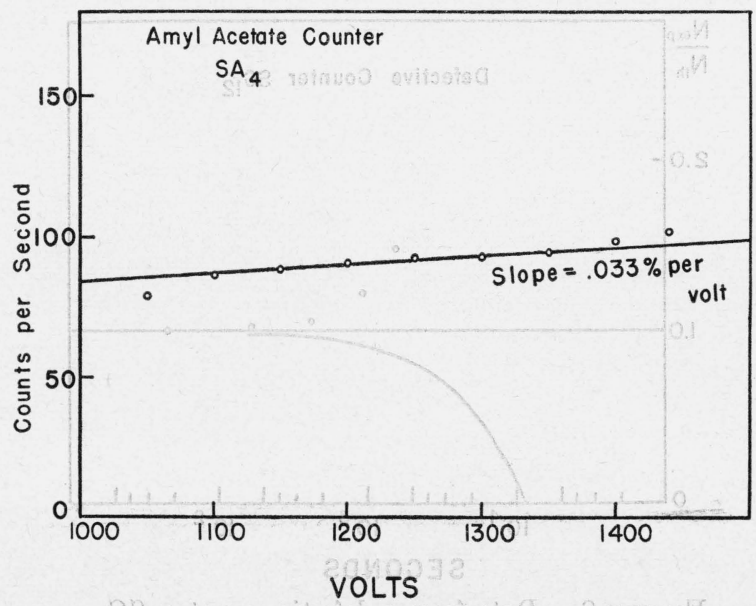

Figure 10.- Voltage characteristic of a typical amyl acetate counter, $S A_{\mathbf{4}}$. A plateau of a range of about 300 volts with satisfactory slope is shown.

ginning at about 1,000 volts. This was repeated with other counters, and they operated in almost identical fashion.

A counter with slope similar to $S A_{4}$ will give results reliable to within 1 percent if the high voltage is stable to within 10 volts. Actually it is readily possible to hold this voltage within closer limits. When counting random pulses, at least 10,000 counts must be counted

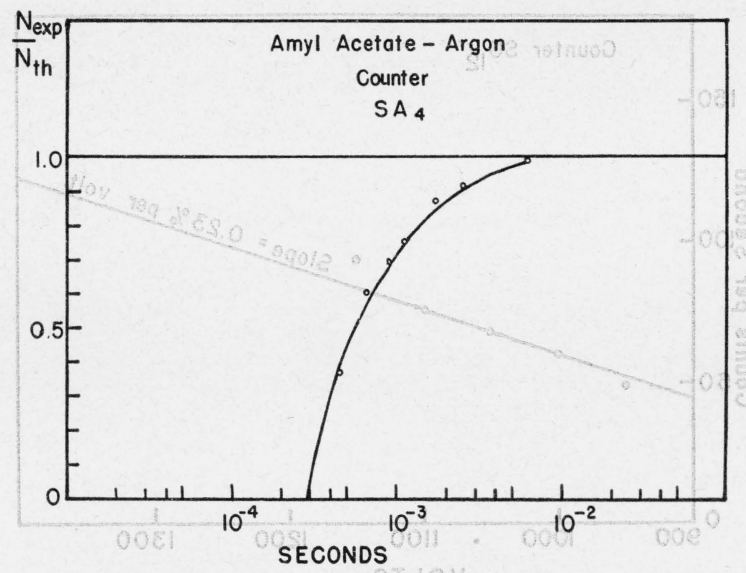

FiguRe 11.-Data for typical counter, $S A_{4}$.

A distribution of intervals in good agreement with the theoretical for a random distribution is shown. 
to obtain an accuracy of 1 percent. Therefore, in many applications this factor places the actual limit on the precision of the results.

From these experiments it can be seen that very satisfactory counters can be prepared using either alcohol or amyl acetate vapor, and argon as a filling medium. Difficulties experienced with these counters arise almost exclusively from failure to secure a mixture of gas and vapor of proper proportions. Following a very simple procedure, every counter prepared can be depended upon to work well, and when the same pressures are used the counters will have very nearly identical characteristics. This places the preparation of this type of Geiger-Müller counter beyond the "Chinese medicine" stage mentioned by some writers. It also makes available a very useful form of counter which requires only the simplest and most direct form of amplifier for its use. The self-quenching properties of these counters also insure that the pulses observed with them originate in the counter tube and not in some auxiliary part of the circuit. It should be emphasized that the authors' experience clearly indicates that no special treatment of the barrel or central wire is required to make a successful counter. All counters constructed to date have been made up with materials as taken from the stockroom. Copper, aluminum, and steel have been used for the barrels and steel and tungsten, for the central wires, with no noticeable difference in performance which could be traced to the materials or construction.

This work is part of a program undertaken at the National Bureau of Standards to investigate reliable methods for the preparation of Geiger-Müller counters. One aim of this investigation is to make possible the direct comparison of counter measurements made in different laboratories through standardization of the tube counters themselves.

Washington, September 14, 1942. 